\title{
AMINO ACID SEQUENCE HOMOLOGY BETWEEN HUMAN AND BOVINE LOW MOLECULAR WEIGHT FOLATE BINDING PROTEIN ISOLATED FROM MILK
}

\author{
by \\ IB SVENDSEN \\ Carlsberg Laboratory, Department of Chemistry \\ Gamle Carlsberg Vej 10, DK-2500 Copenhagen Valby \\ and \\ STEEN INGEMANN HANSEN, JAN HOLM, JØRGEN LYNGBYE \\ Department of Clinical Chemistry, Central Hospital \\ DK-3400 Hillerød
}

Keywords: Folate, amino acid sequence, human milk

The partial amino acid sequence of a low molecular weight folate binding protein from human milk has been determined from $\mathrm{N}$-terminal sequencing of the native protein and isolated cyanogen bromide fragments. A molecular weight of approximately 30,000 is estimated on the basis of amino acid composition and sequence homology with the low-molecular weight folate binder isolated from cow's milk. Preliminary studies with a large molecular weight folate binding protein have shown that the N-terminal amino acid sequence is identical with that of the low molecular binder.

\section{INTRODUCTION}

High molecular weight (MW 100,000 200,000 ) and low molecular weight (MW 25,00 $-40,000$ ) proteins, which specifically bind folate are present in various body fluids and tissues $(4,10)$. Low molecular weight folate binding proteins (FBP) have been isolated from cow's milk $(6,7,8)$, goat's milk $(5)$ and human milk $(1,3,9)$ which in addition contains a high molecular weight FBP $(1,3,9)$. In a previous publication we have described the isolation and partial characterization of the low molecular weight FBP iso- lated from cow's milk (8) and including part of the amino acid sequence. This has now been extended to approximately $95 \%$ of the total sequence (to be published). Parts of these unpublished observations are included in the present paper.

The folate binding proteins present in human milk have until recently only been isolated in quantities insufficient for amino acid sequence studies to be performed. These difficulties have now been overcome (3) to the extent that a par- 
tial characterization in terms of amino acid composition and sequence of the low molecular weight FBP could be performed. The results of this investigation are given in the present paper and a comparison is made with bovine FBP.

\section{MATERIALS AND METHODS}

\subsection{Materials}

Unpasteurized human milk was obtained from Childrens Hospital "Fuglebakken", Copenhagen, and kept at $-18^{\circ} \mathrm{C}$ until used. All chemicals used in the amino acid sequence studies have been described in ref. (8).

\subsection{Methods}

High and low molecular weight FBP were prepared from Triton X-100 solubilized milk as described in (3) by a combination of ion-exchange chromatography on CM-Sepharose CL-6B in the presence of Triton $\mathrm{X}-100$ and affinity chromatography on a methotrexate-AH-Sepharose
4B column desorbed with a $\mathrm{pH}$-gradient. The purity of the low molecular weight FBP was ascertained by $\mathrm{N}$-terminal amino acid sequence analysis on a Beckmann sequenator. Amino acid analysis, carbohydrate determination and amino acid sequence determination were performed as described in (8). Reduction with mercaptoethanol, alkylation with 2-vinylpyridine and cleavage with cyanogen bromide have also been described previously (8).

\section{RESULTS AND DISCUSSION}

\subsection{Amino acid composition}

Due to the limited amount of FBP available a rigourous determination of the amino acid composition was excluded. Only a single 24 hours hydrolysis was made - the result of which is shown in Table I. Column 2 gives the actual amino acid composition determined in nanomoles. The third column gives the composition on a molar basis assuming four methionine

Table I

Amino acid composition of folate binding protein (FBP) from human and bovine milk.

\begin{tabular}{|c|c|c|c|c|}
\hline & & moles $^{2)}$ & moles $^{3)}$ & moles ${ }^{4)}$ \\
\hline Amino acid & nmoles') & moles FBP & moles FBP & moles FBP (cow) \\
\hline Aspartic acid & 10.59 & $24.9(25)$ & 25 & $24.0(24)$ \\
\hline Threonine & 5.21 & $12.3(12)$ & 10 & $14.0(14)$ \\
\hline Serine & 6.89 & $16.2(16)$ & 16 & $22.6(23)$ \\
\hline Glutamic acid & 12.75 & $30.0(30)$ & 29 & $26.0(26)$ \\
\hline Proline & 7.36 & $17.3(17)$ & 14 & $18.9(19)$ \\
\hline Glycine & 5.53 & $13.0(13)$ & 12 & $14.0(14)$ \\
\hline Alanine & 7.58 & $17.8(18)$ & 18 & $14.2(14)$ \\
\hline 1/2-cystine & 6.51 & $15.3(15)$ & n.d. & $11.7(12)$ \\
\hline Valine & 4.36 & $10.3(10)$ & 8 & $11.6(12)$ \\
\hline Methionine & 1.70 & $4.0(4)$ & 5 & $2.7(3)$ \\
\hline Isoleucine & 2.54 & $6.0(6)$ & 6 & $7.2(7)$ \\
\hline Leucine & 4.74 & $11.2(11)$ & 11 & $12.2(12)$ \\
\hline Tyrosine & 5.42 & $12.7(13)$ & 8 & $11.4(11)$ \\
\hline Phenylalanine & 6.58 & $15.5(16)$ & 17 & $12.0(12)$ \\
\hline Histidine & 6.63 & $15.6(16)$ & 17 & $8.3(8)$ \\
\hline Lysine & 6.36 & $15.0(15)$ & 10 & $12.3(12)$ \\
\hline Arginine & 5.99 & $14.1(14)$ & 14 & $13.7(14)$ \\
\hline Tryptophan & n.d. & & n.d. & (8) \\
\hline
\end{tabular}

1) 24 hours of hydrolysis. Uncorrected for loss of Thr and Ser.

2) Based on 4 methionine residues per mole of protein.

3) Recalculated from (1) on the basis of 18 alanine residues per molecule of protein.

4) From (8). The numbers in brackets are nearest intergers.

n.d. $=$ not determined. 
N-terminal of FBP and cyanogen bromide II fragment:

1

10

Human: Ile-Ala-Trp Ala-Arg-Thr-Glu-Leu-Leu-Asn-Va1- ? -Met-Asn-Ala

Bovine: Ala-Gin-Ala-Pro-Arg-Thr-Pro-Arg-Ala-Arg-Thr-Asp-Leu-Leu-Asn-Val-Cys-Met-Asp-Ala-

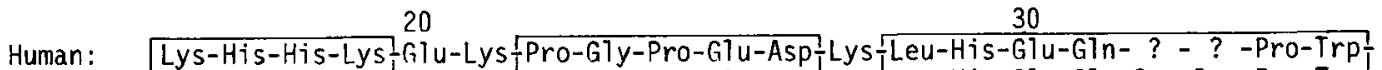

30

Bovine: Lys-His-His-Lys Ala-Glu-Pro-Gly-Pro-Glu-Asp-Ser-Leu-His-Glu-Gin-Cys-Ser-Pro-TrP-

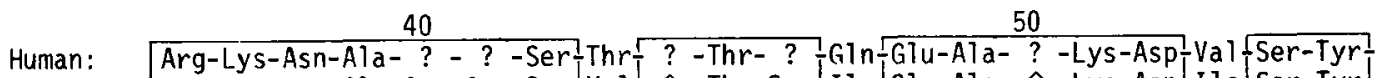

Bovine: $\quad$ Arg-Lys-Asn-Ala-Cys-Cys-Ser-Val $\dot{\imath}-$ Thr-Ser-Ile

60

Human: Leu-Tyr-Arg-Phe-Asn-

Bovine: Leu-Tyr-Arg-Phe-Asn-

Cyanogen bromide I fragment:

Human: Ala-Pro-Ala-Cys-Lys-Arg-His-Phe-Ile-Gln-Asp-Thr-Cys-Leu-Tyr-Glu- ? -Ser-Pro-AsnBovine: Glu Pro-Ala-Cys-Lys-Arg-His-Phe-Ile-Gln-Asp-Thr-Cys-Leu-Tyr-Glu-Cys-Ser-Pro-Asn-

Human: Leu-Gly-Pro ? Ile-GIn-Gln-Val-Asp-Gln-Ser- ? TArg-Lys-Glu-Arg-Val-Leu-Asn-ValBovine: Leu-Gly-Pro-Trp-Ile-Arg-Glu-Val-Asn-GIn-Arg-Trp-Arg-Lys-GIu-Arg-Val-Leu-Gly-Val-

C-terminal cyanogen bromide fragment:

Human: $\quad$ Trp-Phe-Asp-Pro-Ala-GIn-Gly-Asn-Pro-Asn-Glu-Gln-Val-Ala

Bovine: $\quad$ Trp-Phe-Asp-Pro $-P h e-G l n-G 1 y-A s n-P r o-A s n-G 1 u_{-} G 1 U_{-V}$ Val-Ala

Figure 1. Amino acid sequence of human low molecular weight folate binding protein (milk) and comparison with the equivalent binder isolated from cow's milk (8).

$\checkmark$ Indicates presence of carbohydrate, ? indicates unidentified position.

residues per molecule. This composition is compared to that determined for FBP from cow's milk (column 5, ref.8). Apart from the large deviation in the contents of Ser and His, no great differences are found between the two proteins considering that the composition of human FBP is based on a single analysis. The high content of His in human FBP may in part be due to traces of Tris-buffer, which is ninhydrin reactive and coelutes with $\mathrm{His}$ on the Durrum amino acid analyser.

A comparison is also made in Table $I$ to a recently published amino acid composition of a low molecular weight FBP (column 4) isolated from human milk (1) which probably is identical to the one described in the present paper. As published in (1) the amino acid composition appeared to be quite different from the one determined by us. However, different ways of calculation were used. Ours was based on 4 methionine residues per molecule and that of (1) was based on the binding of 1 mole of folate per mole of protein. If the date of (1) are recalculated on the basis of 18 alanines per mole of protein as determined by us a close agreement is found when it is considered that both determinations are based on a single 24 hours hydrolysis.

\section{2. $\mathbf{N}$-terminal amino acid sequence}

20 nmoles of human FBP were sequenced from the $\mathrm{N}$-terminus through 60 residues. The result is shown in Figure 1 which in addition includes a comparison with the sequence of bovine FBP. No $\mathrm{PTH}$-amino acids were observed in positions 12 , 
$32,33,40,41,46$, and 50 . The FBP was added to the cup with intact disulfide bridges and comparison with bovine FBP suggests that positions $12,32,40$, and 41 are occupied by cystine residues. We have strong indications for positions 44 and 50 to be glycosylated in bovine FBP (unpublished results), therefore we also assume these positions to have glycosylated amino acid residues in the human FBP. The vacant positions 33 and 46 may also contain glycosylated residues. In bovine FBP a Ser is observed in both positions and it is tempting to suggest that these positions similarly are occupied by glycosylated serine or threonine residues in human FBP.

As expected a high degree of homology between the N-termini of the two folate binding proteins was observed. As seen in Figure 1 the human FBP is shorter by five residues than bovine FBP. When the $\mathrm{N}$-terminal sequence of bovine FBP was determined (8) two chains were actually observed: one starting as shown in Figure 1 and another (10\% of total) with Ala(4)$\operatorname{Arg}(5)-\operatorname{Thr}(6)$ (using the nomenclature of Figure 1) indicating that proteolytic cleavage had taken place, probably by trypsin or a trypsin-like protease. Although one chain only was sequenced in human FBP it is possible that proteolytic cleavage has taken place also in this case. An Arg may well have preceeded the $\mathrm{N}$-terminal Ile making the Arg-Ile bond vulnerable for tryptic cleavage.

The similarity of the two sequences is further emphasized by the observation that most of the differences are compatible with a change of only one base per codon triplet. A further verification of the homology between human and bovine FBP was obtained from sequence studies on the peptides obtained by cleavage with cyanogen bromide.

\subsection{Amino acid sequences of cyanogen bromide peptides}

From the study of bovine FBP CNBr-peptides it was known that the C-terminal $\mathrm{CNBr}$-fragment did not contain disulfide bridges and consequently would separate from the other $\mathrm{CNBr}$ fragments by gelfiltrating. This strategy was adopted for human FBP, 20 nmoles of which was cleaved with the disulfide bridges intact and subsequently gelfiltered on a Biogel P 30 column. A

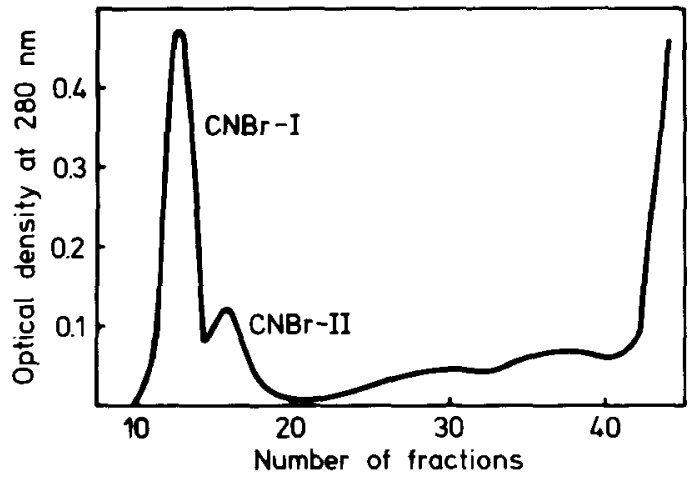

Figure 2. Separation of cyanogen bromide fragments of human low molecular weight folate binding protein on a Biogel P $30(0.9 \times 60 \mathrm{~cm})$ column equilibrated and eluted with $30 \%$ acetic acid.

Flow-rate $4 \mathrm{ml}$ hour $^{-1}$. Fraction of $0.8 \mathrm{ml}$ were collected.

pure peptide separated from the main peak and it was sequenced 14 steps. As seen in Figure 1 a close similarity with the $\mathrm{C}$-terminal $\mathrm{CNBr}$-fragment of bovine FBP was observed the only differences being an Ala (human) instead of a Phe (bovine) in position five, and a Gln (human) instead of Glu (bovine) in position 12 .

The front peak from gel filtration of the $\mathrm{CNBr}$-cleavage was lyophilized, reduced with mercaptoethanol and treated with vinylpyridine. The reaction product was applied directly to the same Biogel P 30 column as above and gel filtration resulted in five peaks (Figure 2 ) of which the third and the last one did not contain peptides.

Peak 1 ( $10 \mathrm{nmoles}$ ) sequenced for 40 cycles and the result is shown in Figure 1. Again, a close similarity with the sequence of $\mathrm{CNBr}-\mathrm{I}$ of bovine FBP was observed (only six amino acid residues were at variance).

The sequence of peak 2 was followed 22 steps and was identical with the sequence Asn (14) to $\operatorname{Trp}(35)$ already determined on the intact protein (Figure 1). The expected homology with $\mathrm{CNBr}$ II of bovine FBP was thus demonstrated.

\subsection{Carbohydrate determination}

Human FBP is a carbohydrate containing protein which was indicated by the presence of a glucosamine peak. No rigourous determination of the content of carbohydrates has been attempted. Removal of FBP from the sequenator 
cup, by $2 \times 500 \mu l 30 \%$ acetic acid, at the end of the $\mathrm{N}$-terminal sequence determination followed by a determination of neutral sugars (2) indicated a content of $3 \%$.

\subsection{Molecular weight determination}

The molecular weight of human FBP was estimated in two ways. Gel filtration on Ac A44 with Triton X-100 $\lg \times 1^{-1}$ in the elution buffer gave a molecular weight of 27,000 (3). Under similar conditions Antony et al. (1) found 30,500 and 43,000 respectively. In addition, these authors found a molecular weight of 40,000 from gel filtration on Sephadex G200. On the other hand, WAXMAN and SCHREIBER (9) determined a molecular weight of 26,500 by gel filtration on Sephadex G200.

We also estimated the molecular weight from the amino acid composition, assuming 4 methionine residues per mole of FBP, to be 29,000 and if $3 \%$ carbohydrate is included, a molecular weight of approximately 30,000. AnTONY et al. (1) have also estimated the molecular weight from amino acid composition and carbohydrate content assuming the binding of $1 \mathrm{~mol}$ folate per mole of FBP. They arrived at 21,300 for the amino acid part and 6,000 (or $22 \%$ ) for the carbohydrate or a total of approximately 27,000 .

As demonstrated above great confusion exists with respect to the true molecular weight of human FBP a feature which was also noted with bovine FBP. Part of the confusion can be attributed to the inherent difficulty in obtaining reliable values for glycoproteins by gel filtration and SDS-PAGE. A molecular weight based on amino acid and carbohydrate composition therefore seems to be preferable. Although fair agreement is found between our studies and those of ANTONY et al. (i) for the total molecular weight, that is, the sum of amino acids and carbohydrates the estimation of each of the components vary greatly (vide supra). We believe that the amino acid composition determined by us is nearly correct and base this assumption on the amino acid homology between human and bovine FBP. Since the publication of the partial amino acid sequence of bovine FBP (8) we have almost finished the complete sequence $(95 \%)$ from which a molecular weight of $\sim 29,000$ is calculated which is similar to the one obtained for the human FBP (3). Furthermore, the sequence homology between the human and bovine FBP extends all the way to the $\mathrm{C}$-terminal 30 residues (see Figure 1). We therefore consider the two sequences to have approximately the same length (except for five residues at the $\mathrm{N}$-terminus) which is compatible with the calculated molecular weight. Although deletion of a large amino acid sequence somewhere in the molecule cannot be ruled out we consider this to be a remote possibility.

It should be pointed out that our estimated total molecular weight of 30,000 is a minimum molecular weight, since our estimation of the carbohydrate content may be too low.

\subsection{High molecular weight FBP}

In a recent publication by ANTONY et al. (1) it was suggested that the low molecular weight human FBP was obtained from the high molecular weight FBP by proteolytic cleavage, since the two binders were immunological indistinguishable. Preliminary studies by us with a high molecular weight FBP preparation $10 \%$ contaminated with low molecular weight FBP (judged by gel filtration) have shown that the two binders have identical $\mathrm{N}$-terminal amino acid sequence for 39 cycles. This finding strongly suggests that the two binders have the same genetic origin and that the low molecular weight binder is a result of proteolytic cleavage. The alternative suggestion by ANTONY et al. (1) that the two binders are coded for by two different genes that arose from a common ancestral gene cannot be excluded, but the identity of the $39 \mathrm{~N}$-terminal residues does not point towards this alternative.

The demonstrated homology between the amino acid sequences of human and bovine FBP is encouraging for the planning of future studies of this protein, especially those studies concerned with the structure-function relationships. Results obtained with bovine FBP, which is obtainable in much larger quantities and which is easier to prepare, will most likely be valid also for human FBP.

\section{ACKNOWLEDGEMENTS}

We wish to thank Professor MARTin OtTesen for critical review of the manuscript, and Mrs. Bodil Corneliussen, Lone Sørensen, Jytte 
Rasmussen and Solveig NordLunde for expert technical assistance. The study received financial support from the Danish Foundation for The Advancement of Medical Science (57/80).

\section{REFERENCES}

1. Antony, A.C., C.S. Utley, P.D. Marcell \& J.F. KolHoUSE: Isolation, characterization, and comparison of the solubilized particulate and soluble folate binding proteins from human milk. J.Biol.Chem. 257, 10081-10089 (1982)

2. Dubois, M., K.A. Gilles, J.E. Hamilton, P.A. REBERS \& F. SMITH: Colorimetric method for determination of sugars and related substances. Anal.Chem. 28, 350-356 (1956)

3. Hansen, S.I., J. Holm \& J. Lyngbye: Highaffinity binding of folate to a small and large molecular size protein from human milk. Proc. in Chemistry and Biology of Pteridines, in press. Seventh International symposium on Pteridines and folic acid derivative. St. Andrews, Scotland 21-24 September (1982). J.A. Blair, ed., Walter de Gruyter and Co., Berlin.

4. Rothenberg, S.P. \& M. DACosta: Folate binding proteins and radioassay for folate. Clinics in Haematology 5, 569-585 (1976)
5. Rubinoff, M., C. Schreiber \& S. Waxman: The isolation and characterization of the folate binding protein from goat milk. FEBS Lett. 75, 244-248 (1977)

6. Salter, D.N., J.E. Ford, K.J. Scott \& P. ANDREWS: Isolation of the folate-binding protein from cow's milk by the use of affinity chromatography. FEBS Lett. 20, 302-306 (1972)

7. Salter, D.N., K.J. Scott, H. Slade \& P. ANDREWS: The preparation and properties of folate-binding protein from cow's milk. Biochem.J. 193, 469-476 (1981)

8. Svendsen, I., B. Martin, T.G. Pedersen, S.I. HANSEN, J. Holm \& J. LyngBye: Isolation and characterization of the folate-binding protein from cow's milk. Carlsberg Res. Commun. 44, 89-99 (1979)

9. WaXman, S. \& C. Schreiber: The purification and characterization of the low molecular weight human folate binding protein using affinity chromatography. Biochemistry $14,5422-$ 5428 (1975)

10. Wayman, S., C. Schreiber \& M. Rubinoff: The significance of folate binding proteins in folate metabolism. Adv. Nutritional Res. 1, 5576 (1977) 